\title{
Article
}

\section{Systematic problems with using dark matter simulations to model stellar halos}

Bailin, Jeremy, Bell, Eric F., Valluri, Monica, Stinson, Greg S.,
Debattista, Victor P, Couchman, H. M. P. and Wadsley, James Available at http://clok.uclan.ac.uk/12505/

Bailin, Jeremy, Bell, Eric F., Valluri, Monica, Stinson, Greg S., Debattista, Victor P ORCID: 0000-0001-7902-0116, Couchman, H. M. P. and Wadsley, James (2014) Systematic problems with using dark matter simulations to model stellar halos. The Astrophysical Journal, 783 (2). p. 95. ISSN 0004-637X

It is advisable to refer to the publisher's version if you intend to cite from the work. http://dx.doi.org/10.1088/0004-637X/783/2/95

For more information about UCLan's research in this area go to http://www.uclan.ac.uk/researchgroups/ and search for <name of research Group>.

For information about Research generally at UCLan please go to http://www.uclan.ac.uk/research/

All outputs in CLoK are protected by Intellectual Property Rights law, including Copyright law. Copyright, IPR and Moral Rights for the works on this site are retained by the individual authors and/or other copyright owners. Terms and conditions for use of this material are defined in the policies page.

\section{CLoK}

Central Lancashire online Knowledge www.clok.uclan.ac.uk

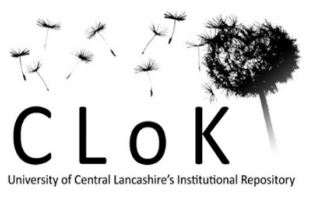




\title{
SYSTEMATIC PROBLEMS WITH USING DARK MATTER SIMULATIONS TO MODEL STELLAR HALOS
}

\author{
Jeremy Bailin ${ }^{1}$, Eric F. Bell ${ }^{2}$, Monica Valluri ${ }^{2}$, Greg S. Stinson $^{3}$, Victor P. Debattista ${ }^{4}$, \\ H. M. P. COUCHMAN ${ }^{5}$, AND JAMES WADSLEY ${ }^{5}$ \\ ${ }^{1}$ Department of Physics and Astronomy, University of Alabama, Box 870324, Tuscaloosa, AL 35487-0324, USA; jbailin@ua.edu \\ 2 Department of Astronomy, University of Michigan, 830 Dennison Building, 500 Church Street, Ann Arbor, MI 48109, USA \\ ${ }^{3}$ Max-Planck-Institut für Astronomie (MPIA), Königstuhl 17, D-69117 Heidelberg, Germany \\ ${ }^{4}$ Jeremiah Horrocks Institute, University of Central Lancashire, Preston PR1 2HE, UK \\ ${ }^{5}$ Department of Physics and Astronomy, McMaster University, 1280 Main Street West, Hamilton, ON L8S 4M1, Canada \\ Received 2013 April 25; accepted 2014 January 21; published 2014 February 20
}

\begin{abstract}
The limits of available computing power have forced models for the structure of stellar halos to adopt one or both of the following simplifying assumptions: (1) stellar mass can be "painted" onto dark matter (DM) particles in progenitor satellites; (2) pure DM simulations that do not form a luminous galaxy can be used. We estimate the magnitude of the systematic errors introduced by these assumptions using a controlled set of stellar halo models where we independently vary whether we look at star particles or painted DM particles, and whether we use a simulation in which a baryonic disk galaxy forms or a matching pure DM simulation that does not form a baryonic disk. We find that the "painting" simplification reduces the halo concentration and internal structure, predominantly because painted DM particles have different kinematics from star particles even when both are buried deep in the potential well of the satellite. The simplification of using pure DM simulations reduces the concentration further, but increases the internal structure, and results in a more prolate stellar halo. These differences can be a factor of 1.5-7 in concentration (as measured by the half-mass radius) and 2-7 in internal density structure. Given this level of systematic uncertainty, one should be wary of overinterpreting differences between observations and the current generation of stellar halo models based on DM-only simulations when such differences are less than an order of magnitude.
\end{abstract}

Key words: galaxies: formation - galaxies: halos - galaxies: structure - Galaxy: halo - Galaxy: structure methods: numerical

Online-only material: color figures

\section{INTRODUCTION}

While it is now abundantly clear that much of the mass in the extended outer stellar envelopes of galaxies (stellar halos hereafter) is stripped from dwarf galaxies as they tidally interact with the central galaxy (Majewski et al. 2003; Bullock \& Johnston 2005, hereafter BJ05; Purcell et al. 2007; Bell et al. 2008; McConnachie et al. 2009; Cooper et al. 2010, hereafter C10; Xue et al. 2011; Ibata et al. 2014), a number of questions remain. Is all of the halo mass accreted (BJ05, C10; Rashkov et al. 2012), or are substantial fractions kicked up from the stellar disk (called in situ; Kazantzidis et al. 2008; Zolotov et al. 2009; Font et al. 2011) or formed within satellites after they have been accreted (Tissera et al. 2013; Valluri et al., in preparation)? How much variation is expected from halo to halo (BJ05, Bell et al. 2008; Font et al. 2011)?

Many studies focus on these issues by comparing observations of stellar halos with models of stellar halo formation in a cosmological context (Bell et al. 2008, 2010; Helmi et al. 2011; Xue et al. 2011; Schlaufman et al. 2012; Monachesi et al. 2013). In most cases, cognizant that much of the stellar halo is accreted, comparisons are made to models in which the stellar halo is constituted only of accreted material: this simplifies interpretation, and any major discrepancies between the observations and such models could signal that this assumption is incorrect, giving insight into other possible modes of halo formation.

A generic practical problem that one encounters when creating a theoretical numerical model of stellar halos is resolution. Although stellar halos are potentially rich with signposts of the hierarchical galaxy formation process, they contain a very small fraction of the total stellar mass in a galaxy. For example, the stellar halos of the Milky Way and similar-mass galaxies account for only $\approx 1 \%-10 \%$ of their stellar content (BJ05; Bailin et al. 2011; Ibata et al. 2014). In order to resolve the tidal streams that constitute the halo and provide observational tests of the hierarchical merging paradigm, hundreds of thousands of particles must be used within the halo itself. Simulating the entire galaxy self-consistently at this resolution would then require hundreds of millions of particles, a task that would require tens of millions of CPU hours per galaxy with current algorithms and hardware. The problem is exacerbated by the stochastic nature of merger histories, which result in factors of several galaxy-to-galaxy variation in stellar halo properties even at a given galaxy mass (e.g., Purcell et al. 2007) and requires performing a significant number of these simulations in order to make robust predictions.

The common solution to this problem is to not simulate the entire galaxy self-consistently. Pure $N$-body simulations are much faster than full hydrodynamic simulations at a given resolution, at the expense of not including any non-gravitational processes (e.g., C10; Libeskind et al. 2011, hereafter L11). Furthermore, if the main body of the galaxy is replaced by an analytic potential, the number of particles gets dramatically reduced and it becomes feasible to simulate the halo at high resolution in a reasonable length of time (e.g., BJ05). In these pure $\mathrm{N}$-body methods $(\mathrm{C} 10, \mathrm{~L} 11)$, dark matter (DM) particles must be labeled ("painted") to represent the stellar component in lieu of having a self-consistent method of generating stars. Other tactics that have been taken are to use lower resolution self-consistent simulations where the internal structure of the halo is poorly resolved (e.g., Font et al. 2011; in low resolution simulations, unresolved physical processes may also have a significant impact on the derived halo properties, although in 
the case of Font et al. 2011 the authors have tested that their conclusions are robust to a factor of two change in spatial resolution), or to use semi-analytic prescriptions that have explicitly no internal structure but only predict the total amount of halo material (Purcell et al. 2007).

However, there are important differences between the expectations of these different approaches. The difference that inspired this work was the factor-of-two difference in the degree of substructure predicted by BJ05 and $\mathrm{C} 10$. As described by Helmi et al. (2011) and Bell et al. (2008), these models predict an amount of substructure (measured using the root mean square (rms) of the model around a smooth halo profile, divided by the total number of stars) different by a factor of two or more from each other, in the sense that the $\mathrm{C} 10$ models have considerably more substructure than those of BJ05 (Schlaufman et al. 2012 find a similar difference between the BJ05 and Rashkov et al. 2012 models). The $\mathrm{C} 10$ models also have more substructure than the observations; Helmi et al. (2011) interpreted this as a model-data discrepancy signaling the need for halo stars to form in situ; we interpret this as a model-model discrepancy signaling the need to better understand why two seemingly reasonable models should disagree so significantly. One potential point of distinction between the models was that, while BJ05 had an analytic potential (and therefore would have been perhaps more likely to have a more structured halo), it included the potential from a disk.

As discussed earlier, the inclusion of baryons in simulations of galaxy formation adds a degree of complexity and computational cost that both reduces the general applicability of the simulations and prohibits the construction of samples of stellar halos that adequately span the range of possible assembly histories. In this work, we use a hydrodynamical simulation from the McMaster Unbiased Galaxy Simulations project (Stinson et al. 2010), which is of sufficiently high resolution that the satellites whose accretion we wish to follow are well resolved, to explore two crucial aspects of the relationship of baryons to DM relevant to stellar halo formation. First, the dissipative formation of a disk changes the potential of the galaxy, enhancing the strength of the tidal field and affecting the orbits of halo stars (Peñarrubia et al. 2010). Second, we wish to explore the importance of the practice of "painting" stars onto DM particles: inasmuch as DM particles have not suffered dissipation, even the most-bound DM particles have orbits that are very likely to be different from realistic stellar orbits, and this would affect the properties of the resulting stellar halos.

The plan of this paper is as follows. In Section 2, we give an overview of the assumptions that have been used in previous work and the models that we will use to test their effects. In Section 3, we provide full details of how the simulations and models are generated. Section 4 contains the results from the different models, and in Section 5 we discuss what these results imply about the influence of the standard assumptions on halo models. Finally, our conclusions are presented in Section 6.

\section{OVERVIEW}

There are two key assumptions that previous halo models have often adopted in order to make the problem tractable, which we label "painting" and "dark matter dynamics."

Painting. All high resolution stellar halo models consist of pure $N$-body simulations that contain only DM particles. In order to predict the properties of the luminous stellar halo, the authors "paint" stellar mass onto certain DM particles, and then measure the properties and structure of these painted particles. The methods used to paint vary: BJ05 resolve each contributing subhalo into $10^{5} \mathrm{DM}$ particles, and then paint the most-bound particles such that the luminosity follows a King profile; L11 has equal-mass DM particles that are painted equally if they are sufficiently deep in the potential well; $\mathrm{C} 10$ use a sophisticated semi-analytic galaxy formation model to determine the expected amount and distribution of star formation within each contributing subhalo and paint stellar masses onto the DM particles so as to contain the appropriate star formation history; and Rashkov et al. (2012) paint the most-bound DM particles within each subhalo equally, but with a stellar mass that varies between subhalos.

Dark matter dynamics. When galaxies form, baryons cool and collapse into a centrifugally rotating disk, whose morphology can then be altered by further accreted material and interactions with other galaxies; these processes do not occur in pure DM simulations, which do not have the capability to radiatively cool. The gravitational potential in which the stars that constitute the stellar halo orbit is therefore different in the real universe than in a pure DM simulation: it is more concentrated, and is flattened in the inner regions due to the disk (Kazantzidis et al. 2004; Bailin et al. 2005; Debattista et al. 2008; Tissera et al. 2010). Different groups have taken different approaches to account for this effect: C10 and Rashkov et al. (2012) use pure $\mathrm{N}$ body cosmological simulations and neglect any changes in the potential due to baryonic physics; BJ05 grow an analytic disk potential inside an analytic growing halo potential; and finally, a particularly interesting approach is that of L11, who compare a full hydrodynamic cosmological simulation to the identical DM-only simulation using the same initial conditions. They find that, when a gravitationalpotential-based painting scheme is adopted (see above), the radial distribution of the stellar halo in both simulations is identical, and therefore argue that the effect of the baryonic physics can be taken entirely into account by the appropriate painting scheme.

These two types of assumptions have remained largely untested, and their effect on the final properties of the predicted stellar halos are therefore unknown. Our goal is to use a set of control simulations in which we vary either the method by which we determine where "stars" lie in the simulation volume or the potential in which the particles orbit. To do this, we compare a full smoothed particle hydrodynamics (SPH) simulation of galaxy formation from the McMaster Unbiased Galaxy Simulations (MUGS; Stinson et al. 2010) with a DMonly simulation of the same initial conditions. We analyze four different models for the stellar halo that is formed:

1. SPH-STARS: the stars that form self-consistently in the SPH simulation that are accreted from satellites,

2. SPH-PAINTED: DM particles in the SPH simulation that are painted to match the mean stellar mass-DM mass relation of satellites in the simulation,

3. SPH-EXACT: DM particles in the SPH simulation that are painted to match the stellar mass of each individual satellite that contributes to the halo, and

4. DM-PAINTED: painted DM particles in the DM-only simulation.

Full details of how each of these models is constructed is given in Section 3.3. Our painting schemes are calibrated using the 
luminous satellites within the SPH simulation. This allows us to directly compare the painted stars with those that form selfconsistently in the simulations, since the same objects should have the same stellar content. The comparison between SPHSTARS and SPH-PAINTED halos isolates the effect of using painted DM particles instead of stars (and the SPH-EXACT halo can be used to determine what aspect of the painting scheme is responsible for any differences), while the comparison between the SPH-PAINTED and DM-PAINTED halos isolates the effect of the baryonic contribution to the gravitational potential.

We emphasize here that although we will use the SPH-STARS halo as the reference model, this is not because we think it is a good approximation to stellar halos in the real universe. Galaxy formation simulations at the resolution of MUGS generically form too many stars by a factor of $\sim 2$ (Stinson et al. 2010), contain too large a fraction of their stellar mass in their spheroid (e.g., MUGS galaxies have a mean bulge fraction of $73 \%$ compared to an observed value of $\sim 40 \%$ for comparableluminosity observed galaxies; Tasca \& White 2011), and cannot resolve the majority of streams that constitute the halo. However, the relative comparison between the models is valid: if we start with the same amount of stellar material in the same satellites, then the stellar halos should be similar if the assumptions we are testing are appropriate.

\section{SIMULATIONS}

\subsection{MUGS}

The simulations we analyze are (1) g15784 from the MUGS (Stinson et al. 2010) and (2) a simulation with the same initial conditions but evolved purely using collisionless $N$-body dynamics, i.e., only with DM. These two simulations provide everything we need to cleanly measure the importance of the assumptions we are testing. The total mass of the galaxy within the virial radius (i.e., including subhalos) is $1.4 \times 10^{12} M_{\odot}$ at $z=0$, of which $1.1 \times 10^{11} M_{\odot}$ is in the form of stars and $1.0 \times 10^{11} M_{\odot}$ is in the form of gas. The simulation uses a $\Lambda$ CDM cosmology with $H_{0}=73 \mathrm{~km} \mathrm{~s}^{-1} \mathrm{Mpc}^{-1}, \Omega_{m}=0.24$, $\Omega_{\Lambda}=0.76, \Omega_{b}=0.04$, and $\sigma_{8}=0.76$ (Spergel et al. 2007). The DM particle mass is $1.1 \times 10^{6} M_{\odot}$ in the SPH simulation and $1.3 \times 10^{6} M_{\odot}$ in the DM-only simulation, ${ }^{6}$ the initial gas particle mass is $2.2 \times 10^{5} M_{\odot}$, and the initial star particle mass is $6.3 \times 10^{4} M_{\odot}$. The gravitational softening is $312.5 \mathrm{pc}$. The visible galaxy that forms at $z=0$ has a prominent disk that can be traced to $10 \mathrm{kpc}$ in gas and $20 \mathrm{kpc}$ in stars, with a scale length of $1.7 \mathrm{kpc}$ and has a bulge-to-total ratio of 0.48 within $25 \mathrm{kpc}$. It is therefore a good analog for an early-type disk galaxy.

Eighty-seven snapshots of the SPH simulation were saved, while 62 snapshots of the DM simulation were saved. DM snapshots were spaced equally in time with $\approx 215$ Myr between snapshots; SPH snapshots exist at all of these times plus some additional times corresponding to convenient redshifts.

\subsection{Merger Trees}

Halos were found in the simulations using Amiga's Halo Finder (AHF; Knollmann \& Knebe 2009), which generates a spatially adaptive mesh on which the density field is measured, and then structures are found in the density field corresponding to a virialized spherical overdensity criterion. Because of the

\footnotetext{
6 The difference is because both simulations contain the same number of DM particles, but in the SPH simulation they account for only $83 \%$ of the total mass instead of the entire mass in the DM simulation.
}

adaptive mesh, structures can be found on different scales, and so AHF generates a hierarchy of halos, subhalos, subsubhalos, etc. Finally, energetically unbound particles are removed from the particle lists corresponding to each halo. The output contains the list of particles associated with each halo in each snapshot.

To follow the evolution of individual halos, we must associate DM halos in successive snapshots. To do this, we compare the list of particle IDs between each pair of halos, and assign halo $h_{i}$ in snapshot $s_{i}$ to be the progenitor of halo $h_{i+1}$ in snapshot $s_{i+1}$ if $h_{i}$ contributes more particles to $h_{i+1}$ than any other halo in $s_{i}$ does. We also must be careful with substructure: AHF can assign particles to multiple halos, and in particular, the particles of a subhalo are usually also members of the parent halo. Therefore, if two subhalos merge within a parent halo, the parent halo may appear to be the progenitor of the merger product, because it contributes all of the particles in both subhalos. We therefore assign the progenitor to be the subhalo whose contribution to $h_{i+1}$ most closely matches the actual number of particles in $h_{i+1}$ in these cases.

Linking together each halo with its successor results in a track, which we consider to be the evolution of an individual object. The merger tree "trunk" is the track that results in the parent halo at $z=0$. A list of the maximum mass each track achieves and the snapshot at which that mass is reached is recorded and used for the particle painting (Section 3.3.2).

\subsection{Model Halos \\ 3.3.1. Accreted Stars}

The SPH-STARS halo model consists of all accreted stars in the full SPH simulation. We define accreted stars as those that are not within the AHF particle list (i.e., outside of the virial radius) of the parent halo trunk at the first simulation output in which they appear, but which appear within the particle list of the parent halo at $z=0$. Note that this neglects stars that form in satellites after they have been accreted into the parent halo, but which are later stripped (Tissera et al. 2013; Valluri et al., in preparation). Although it is theoretically possible for a star particle to form in a satellite outside of the trunk but fall into the parent halo before the next output, outputs are placed closely enough together in time (at most $215 \mathrm{Myr}$ ) that the number of such particles should be small. Each star particle is born with mass $6.3 \times 10^{4} M_{\odot}$, and then loses mass over time due to stellar evolution.

The final stellar halo in the SPH-STARS model contains $3.49 \times 10^{10} M_{\odot}$ in 799248 particles within 1.1 times the virial radius. Because some observational analyses specifically avoid regions with known satellites, we also construct a model halo where we exclude all particles contained in bound substructures that are found by AHF. This "NOSUBS" halo model contains $1.78 \times 10^{10} M_{\odot}$ in 409645 particles.

\subsubsection{Painting Methods}

All halo models aside from SPH-STARS consist of DM particles (in either the DM-only or SPH simulation) that have been assigned a stellar mass. This stellar mass is assigned to merger tree tracks at their maximum-mass snapshot.

SPH-PAINTED. The SPH-PAINTED model is constructed in the full SPH simulation from DM particles that have been painted.

In the SPH simulation, we can measure the actual stellar mass fraction for DM halos at the snapshot where they reach their 


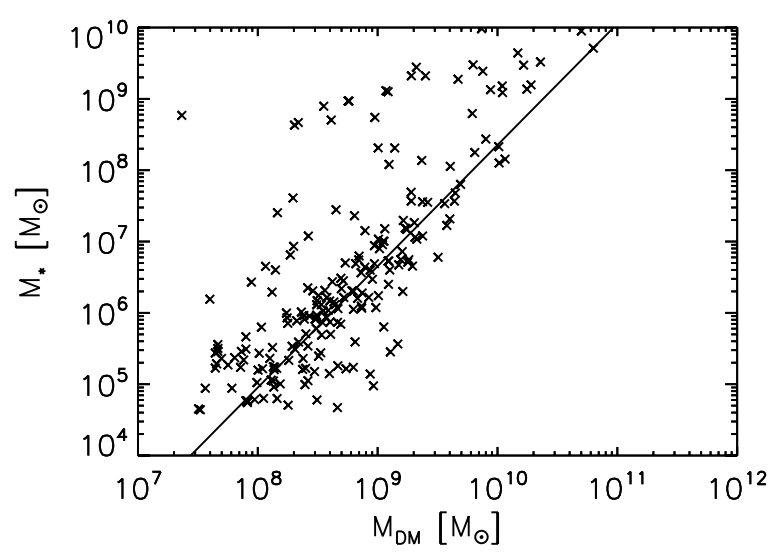

Figure 1. Stellar mass $M_{*}$ of all halos in the MUGS SPH simulation as a function of their dark matter content $M_{\mathrm{DM}}$ at their time of maximum mass. The line is the power law fit given in Equation (1).

maximum mass (Figure 1). The following relationship provides a good fit to the majority of halos:

$$
M_{*}=4.5 \times 10^{6} M_{\odot}\left(\frac{M_{\mathrm{DM}}}{10^{9} M_{\odot}}\right)^{1.7} .
$$

It is possible in the real universe to use the observed satellite galaxy luminosity function and the simulated DM subhalo mass function to estimate the stellar mass fraction satellites must have at infall (which usually corresponds to their time of maximum mass). Studies that do this require there to be a much steeper relationship with lower normalization than what is seen in Equation (1), with power law indices ranging from 2.5 to 3 and normalizations at $10^{9} M_{\odot}$ ranging from $2 \times 10^{3}$ to $2 \times 10^{4} M_{\odot}$ (Koposov et al. 2009; Kravtsov 2010; Rashkov et al. 2012). The SPH simulations, however, produce many more stars in the small objects as a consequence of their well documented tendency to overcool (e.g., Stinson et al. 2010). In order to compare the SPH-STARS and SPH-PAINTED model halos, and therefore determine the effect that particle tagging has on the predicted properties of halos, we must make the progenitors in these models as similar as possible. We therefore match the stellar mass of the progenitor to what forms in the hydrodynamic simulation rather than the stellar content of real galaxies. If the SPH simulations could perfectly reproduce satellite galaxies, then these calibrations would be identical. Equation (1) is therefore the correct stellar mass fraction to adopt for this purpose.

There are a minority of AHF groups (23\%) with an unusually high stellar mass content, with stellar masses greater than or equal to their dark masses. These objects are usually subgroups of larger galaxies, and turn out to be stellar concentrations (e.g., star clusters and spiral arms) that are not independent contributors to the merger history of the main galaxy and should not be painted separately. However, they are assigned a stellar mass that is commensurate with their relatively small dark mass, and are therefore also small and do not contribute significantly to the model halo.

We paint the most-bound ${ }^{7} 1 \%$ of the particles in the halos at the time of maximum mass, and divide the stellar mass evenly amongst these particles. We tested painting different fractions of most-bound particles, and found little qualitative difference

\footnotetext{
7 In determining the "most-bound" particles, we use the total potential plus kinetic energies.
}

in the properties of the resulting stellar halos for values less than $\sim 10 \%$. Our approach is similar to what Rashkov et al. (2012) adopted to generate the halo analyzed by Schlaufman et al. (2012).

The final SPH-PAINTED halo model contains $2.25 \times 10^{10} M_{\odot}$ in 5179 particles, while the NOSUBS version contains $1.58 \times$ $10^{10} M_{\odot}$ in 4043 particles.

DM-PAINTED. The DM-PAINTED model is constructed in the DM-only simulation from painted particles. In order to compare the DM-PAINTED and SPH-PAINTED model halos, and therefore determine the effect that non-gravitational physics has on the predicted properties of stellar halos, we must use the identical painting scheme. We therefore also paint the mostbound $1 \%$ of the particles in the DM halos at the time of maximum mass with a total stellar mass from Equation (1), evenly divided amongst the particles. The AHF groups that are bound by their stellar mass, rather than their DM, are absent in the DM-only simulation, and therefore do not contribute at all to the model halo.

The DM-PAINTED halo model contains $1.45 \times 10^{10} M_{\odot}$ in 4405 particles, while the NOSUBS version contains $1.22 \times$ $10^{10} M_{\odot}$ in 3862 particles.

$S P H-E X A C T$. The SPH-EXACT model, like the SPHPAINTED model, is constructed in the SPH simulation from painted DM particles. However, rather than using Equation (1) to determine the total stellar mass of each progenitor, the exact stellar mass for that progenitor in the SPH simulation is used, as shown in Figure 1. This allows us to ascertain whether any differences between SPH-STARS and SPH-PAINTED are due to the particular method of assigning stellar masses to DM halos, or whether they are generic to the enterprise of painting DM particles.

The SPH-EXACT halo model contains $6.42 \times 10^{10} M_{\odot}$ in 5179 particles, while the NOSUBS version contains $4.49 \times$ $10^{10} M_{\odot}$ in 4043 particles. The choice of painted particles are identical to the SPH-PAINTED model, but they are assigned different stellar masses. The total mass is higher than in SPHPAINTED because the subhalos that do not fall on the best fit relation of Figure 1 scatter systematically high.

\section{HALO STRUCTURE}

\subsection{Overview}

Two-dimensional projected maps of the density of the stellar halo models are shown in Figure 2, which includes all accreted or painted particles, and Figure 3, which excludes those contained in a bound substructure. The pixel size is adaptively expanded from a minimum of $5 \mathrm{kpc}$ per side until there are at least five particles per pixel, so the signal-to-noise per pixel is approximately equal in the low density regions.

We first note that the models unambiguously trace the evolution of the same galaxy: the massive satellites are recognizable in each model at similar locations. It is therefore valid to directly compare the quantitative structure measurements in the different models and be confident that the differences are due to differences in the assumptions of the models, not due to different evolutionary histories.

Second, we note that the models show systematic differences. In particular, it is clear that the global concentration and shape of the stellar halos are different. There also appears to be more structure in the SPH-STARS model than in the SPHPAINTED model, and yet more structure to the DM-PAINTED halo. We will quantify these differences below, which can be 

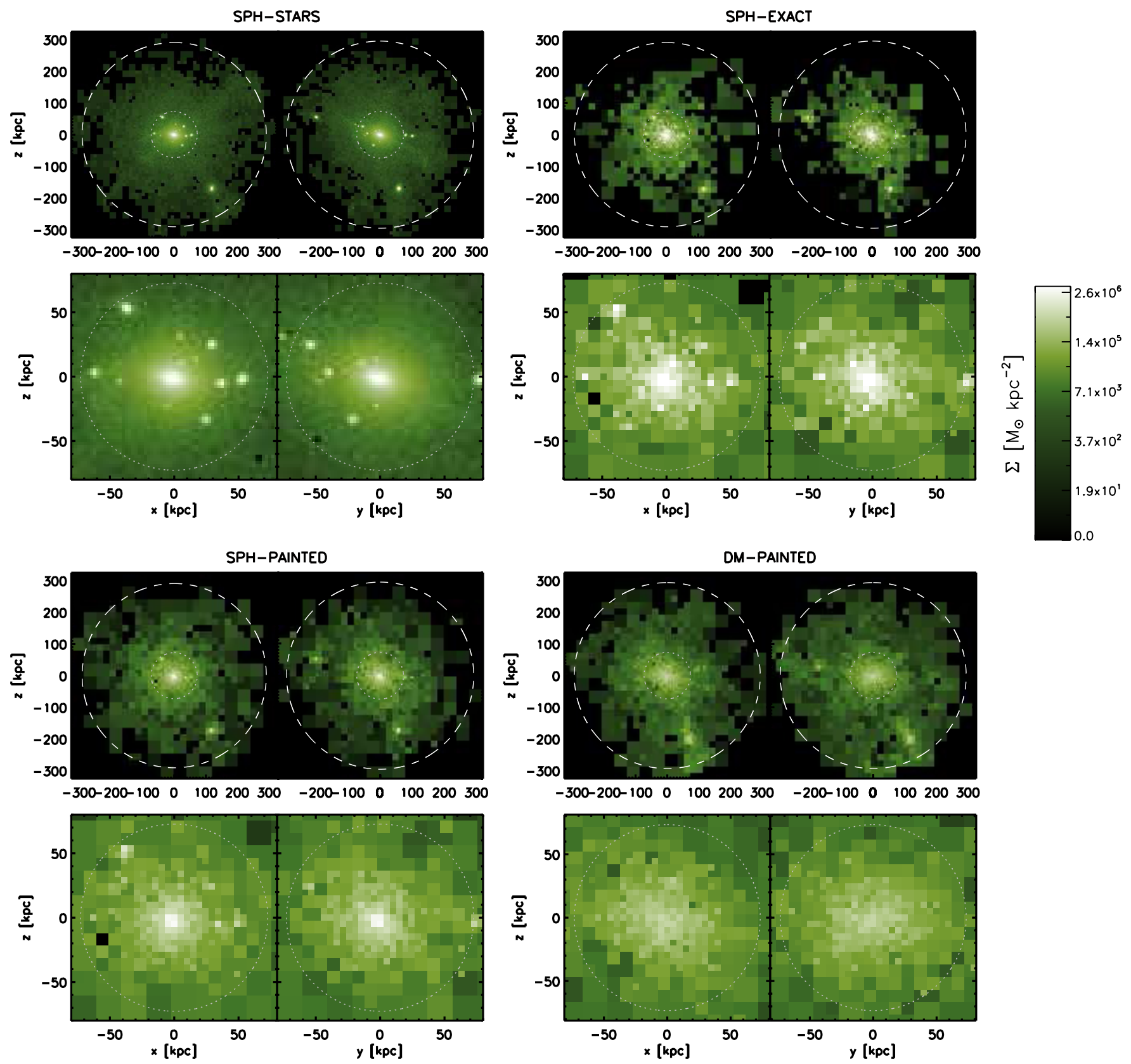

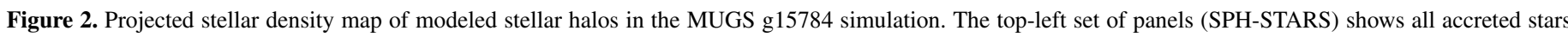

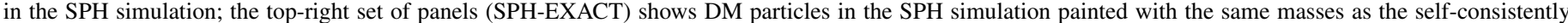

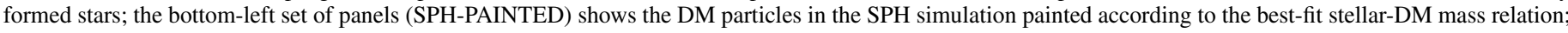

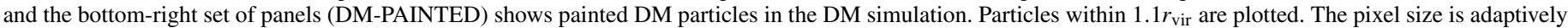

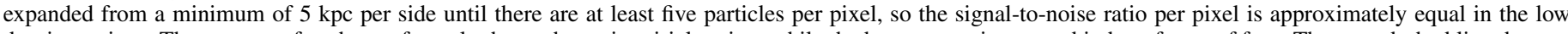

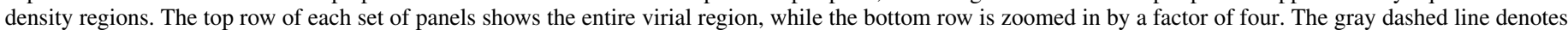
$r_{\mathrm{vir}}$, and the gray dotted line denotes $0.25 r_{\mathrm{vir}}$. The density scale is identical for all panels.

(A color version of this figure is available in the online journal.)

interpreted as an estimate of the magnitude of the systematic errors introduced by the assumptions going into the halo models.

\subsection{Concentration}

To measure the different concentrations of the stellar halo models, we plot their cumulative stellar mass profiles in Figure 4. In the top panel, we include all star particles, while we focus on the bottom panel, where the bound subhalos have been removed. To assess the effect of painting, we compare the SPHSTARS and SPH-PAINTED models. The model containing accreted stars is significantly more centrally concentrated than the painted DM particles in the same simulation-for example, the half-mass radius is more than six times smaller. Because the SPH-STARS model has orders of magnitude more particles, resolution could conceivably be an issue. To assess the impact of the particle number, we have randomly sampled 5179 particles out of the SPH-STARS model to form a new "SPH-STARS (LOWSAMP)" model that has the same number of particles as the SPH-PAINTED model; this is shown in green, and has an identical radial distribution as the full SPH-STARS model. We therefore conclude that the assumption that painted DM particles 

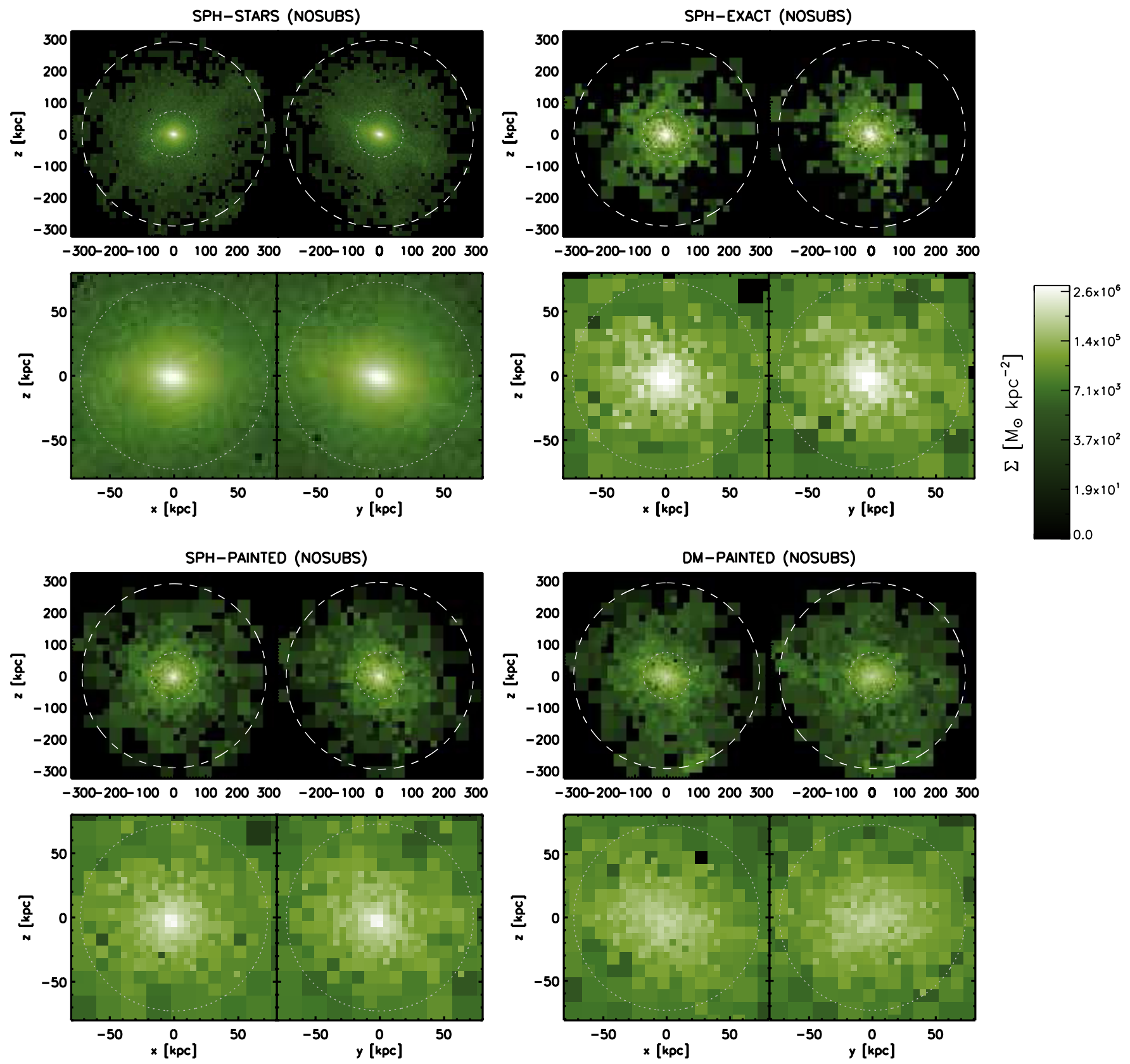

Figure 3. As in Figure 2, but excluding bound subhalos.

(A color version of this figure is available in the online journal.)

follow the same distribution as self-consistently formed stars introduces a large systematic error in the overall concentration of the halo. In the bottom panel, the cumulative mass profiles of power law density distributions $\rho \propto r^{\alpha}$ have been overlaid for various values of $\alpha$. Steep slopes of $\alpha<-3$ appear convex in this plot, while shallow slopes of $\alpha>-3$ appear concave. The SPH-STARS halo is well described by a power law with a slope of $\alpha \sim-3.5$, while the SPH-PAINTED halo transitions from a relatively shallow $\alpha \sim-2$ in the inner regions to a much steeper $\alpha \sim-4$ slope in the outer region.

This general behavior agrees very well with what has been found in the literature. BJ05 find a halo density profile that transitions from a shallow $\alpha \sim-1$ at small radius to $\alpha \sim-3.5$ at large radius, similar to what is seen in the SPH-PAINTED model, which has very similar assumptions. The accreted stars in Font et al. (2011) have a power-law slope of $\sim-3$ at most radii, steepening to -3.5 at large radii, which is not dissimilar to what we find in the SPH-STARS model, and while L11 never quantified the density profile of the self-consistently formed accreted stars in their hydrodynamic simulation, the cumulative mass profile of such stars in their Figure 1 is an excellent match to the analogous SPH-STARS model in our Figure 4.

If this difference is due to assigning an incorrect stellar mass to the accreted halos, then the SPH-EXACT halo should mirror SPH-STARS; if it is due to the choice of DM particles instead of stars, then it should mirror SPH-PAINTED. Figure 4 shows that it is much more similar to SPH-PAINTED; it has the same overall profile shape, and a much more similar halfmass radius. However, it is undoubtedly more concentrated than SPH-PAINTED (the half-mass radius is a factor of two smaller). 


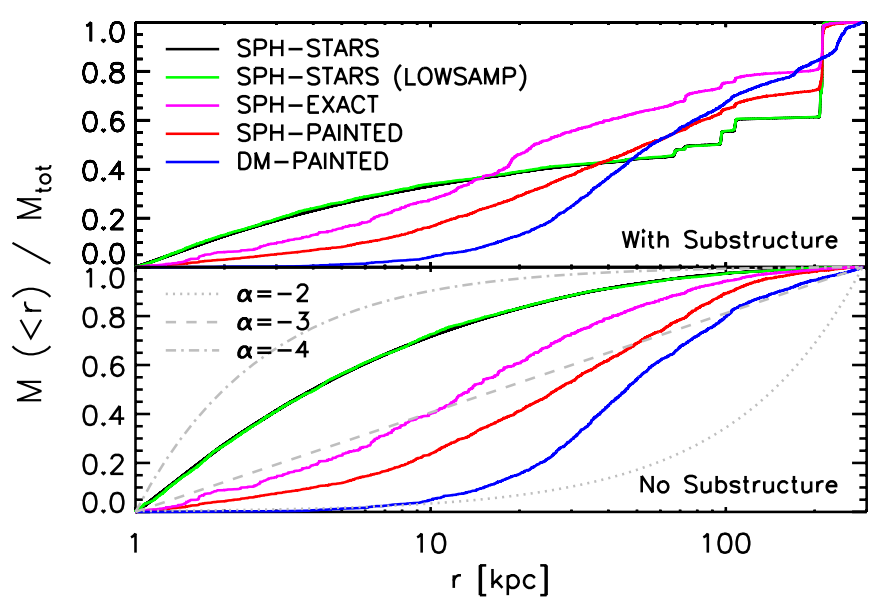

Figure 4. Cumulative radial profile of the stellar mass in the five stellar halo models, starting at $1 \mathrm{kpc}$. The top panel contains substructure, while the substructure has been removed in the bottom panel. The SPH-STARS model is significantly more radially concentrated than the SPH-PAINTED model, which is itself more concentrated than the DM-PAINTED model. The SPH-EXACT halo profile is similar to that of the SPH-PAINTED halo, but slightly more concentrated. The smaller particle number in the LOWSAMP halo has no effect on the concentration, as it lies essentially overtop the SPH-STARS halo. The gray lines in the bottom panel indicate cumulative mass profiles of halos with a power law density profile with slope $\alpha$ between $1 \mathrm{kpc}$ and the virial radius.

This indicates that halos that scatter high in Figure 1 end up at systematically smaller radii, but that the more dominant effect is that painted DM particles are less concentrated than accreted stars.

To assess the impact of the potential, we compare the SPHPAINTED and DM-PAINTED models. We find that they have similar functional forms, but that the SPH-PAINTED model is significantly more concentrated; for example, the half-mass radius is 1.7 times smaller. Therefore, the baryonic contribution to the potential, which is itself more centrally concentrated than the DM, leads to a more centrally concentrated stellar halo.

\subsection{Shape}

Another difference between the models is their global sphericity. Figure 5 shows the shape of the stellar distribution, which has been calculated using the second moment tensor of the stellar mass in an iteratively defined ellipsoidal shell (e.g., Zemp et al. 2011) of width $25 \%$ of the quoted radius. Both the SPHSTARS and SPH-PAINTED halos are somewhat oblate, with $b / a \sim 0.8-1$ and a total flattening rising from $c / a \sim 0.5$ in the inner regions up to 0.8 at the virial radius. On the other hand, model DM-PAINTED, which contains no disk, is very strongly prolate, with $b / a \approx c / a \sim 0.4-0.7$ depending where it is measured. This is not surprising, since the DM halos of simulations with disks are strongly modified by the presence of the disk, becoming less flattened and more oblate, relative to the more flattened prolate DM halos that predominate in pure DM cosmological simulations (e.g., Kazantzidis et al. 2004; Bailin et al. 2005).

\subsection{Substructure}

A key prediction of stellar halo models is the degree of substructure. A rough measurement of this is the variation in the stellar mass density within a shell of a given radius; this is similar to the "sigma/total" measurement used by Bell et al. (2008). Formally, we divide the virial region of the halo into (initially) spherical shells, and then subdivide each shell into angular

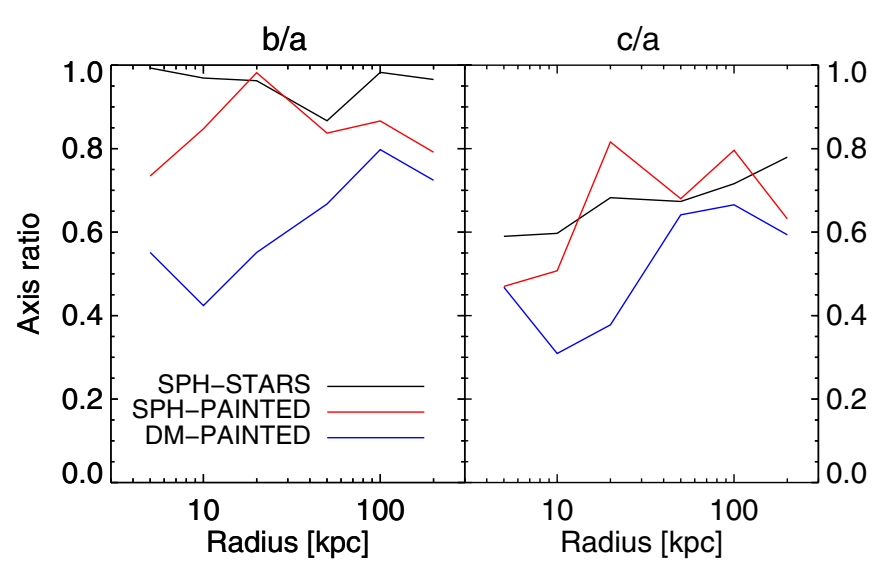

Figure 5. Intermediate (left) and minor (right) axis ratio of each stellar halo model as a function of radius. Shapes are determined iteratively using the second moments of the mass distribution within ellipsoidal shells of width $25 \%$ of the radius, and are plotted at the geometric mean radius of the principal axes. Bound substructures have been removed.

sectors of equal volume. The divisions between these sectors are spaced equally in azimuthal angle $\phi$ and in the cosine of the polar angle $\theta$. We use $N_{\phi}=4$ azimuthal divisions and $N_{\theta}=4$ polar divisions. This probes different physical scales at different radii, and therefore one should not compare the quantitative measurements between radial bins, but rather compare different models at the same radius. We compute the mean stellar mass density of each model within the entire shell $\langle\rho\rangle$, and the rms of the sector-to-sector variation, $\sigma_{\rho}$. There is some contribution due purely to shot noise from the finite number of particles, $\sigma_{\text {shot }}$, the magnitude of which can be determined by noting that the total mass $M$ within a sector is equal to the number of particles $N$ times their mean mass $\langle m\rangle$ :

$$
\begin{aligned}
M & =N\langle m\rangle \\
\sigma_{\text {shot }}^{2} & =\left(\frac{d M}{d N}\right)^{2} \sigma_{N}^{2}+\left(\frac{d M}{d\langle m\rangle}\right)^{2} \sigma_{\langle m\rangle}^{2} \\
& =\langle m\rangle^{2} N+N^{2}\left(\frac{\sigma_{m}^{2}}{N}\right) \\
& =\langle m\rangle^{2} N+N\left(\left\langle m^{2}\right\rangle-\langle m\rangle^{2}\right) \\
& =N\left\langle m^{2}\right\rangle .
\end{aligned}
$$

Technically, this derivation assumes that the mass per sector within each radial bin is independent, while in reality there is an additional constraint that the sum of the masses of the sectors must equal the mass in the shell. However, with 16 sectors, the reduction of one degree of freedom only changes the shot noise by $\approx 3 \%$. We have verified the accuracy of this analytic expression using Monte Carlo experiments. The intrinsic sectorto-sector dispersion $\sigma_{\rho}$ is then the measured rms minus the shot noise $\sigma_{\text {shot }}$, in quadrature.

An additional complication is that the shapes of the halos are different. As noted by Knebe \& Wießner (2006), densities at a given radius can vary by $10 \%-50 \%$ due to the ellipticity of the density distribution, which could dominate the sector-to-sector dispersion if not taken into account. We therefore calculate the shape of the density distribution in a shell of geometric mean radius $30 \mathrm{kpc}$ and width $20 \mathrm{kpc}$, using the method described in Section 4.3, and use these principal axes to define ellipsoidal shells in which to determine $\sigma_{\rho}$. These shells have the same 

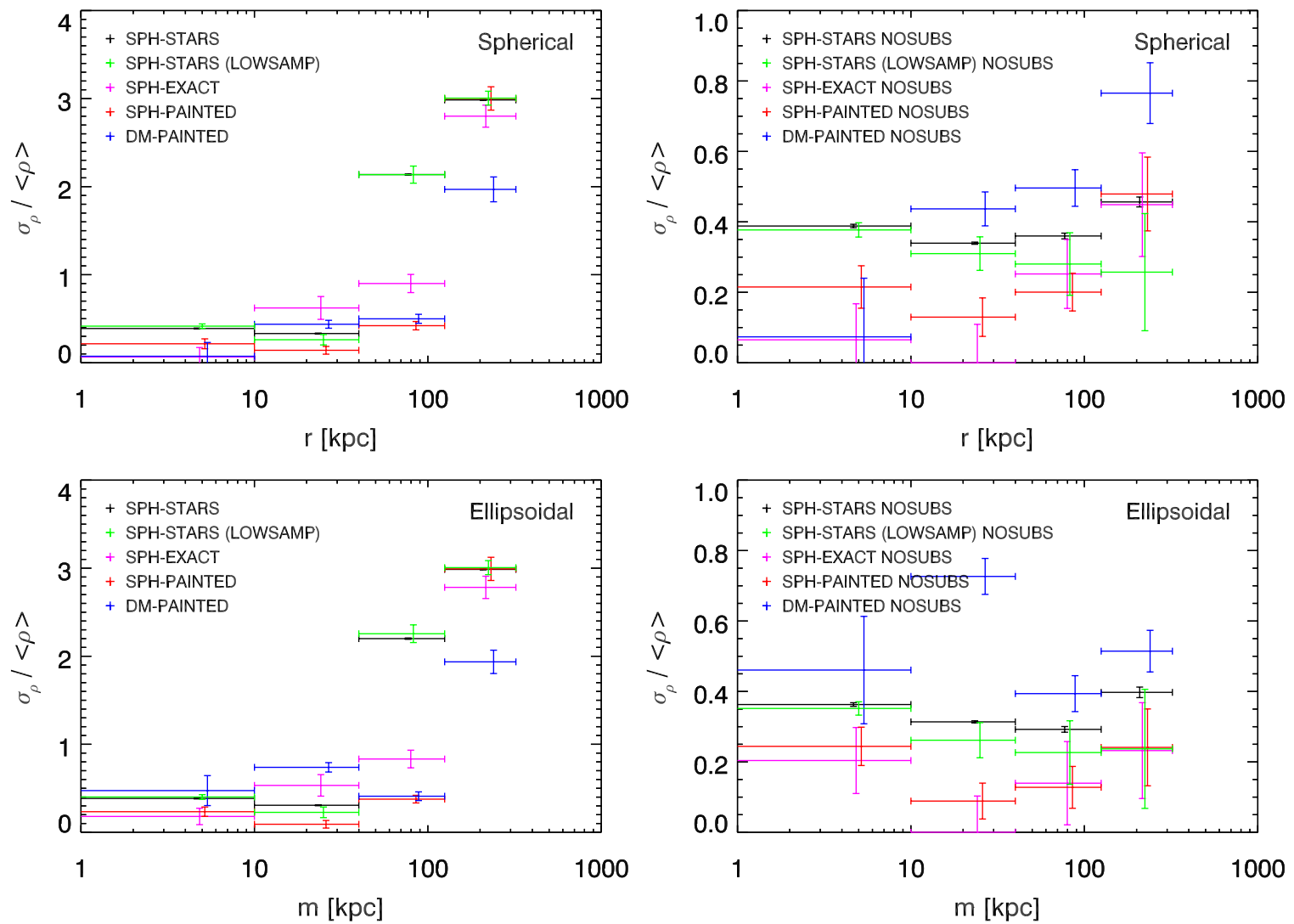

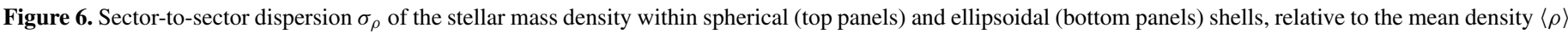

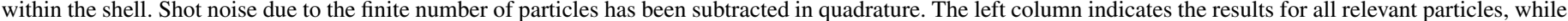

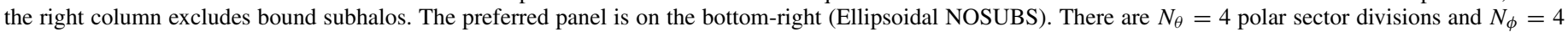
azimuthal sector divisions. Horizontal error bars indicate the radial extent of each bin, while the vertical error bars are the bootstrap error bars in $\sigma_{\rho} /\langle\rho\rangle$.

width and geometric mean radius as the corresponding spherical shells, and therefore the same volume. We also scale the particle coordinates in the principal axis frame by the lengths of the principal axes before we determine which angular sector it belongs to; this ensures that the sectors all have equal volumes regardless of the shape of the ellipsoid.

The amount of structure seen in each halo model is shown in Figure 6. The lefthand plots include all stellar mass, while the bound substructures have been removed in the righthand plots. The top plots show the dispersion within spherical shells, while the bottom plots use the ellipsoidal shells discussed above. The lefthand plots make it apparent that the different models sometimes predict dramatically different amounts of substructure at the same radius; however, there is no clear systematic pattern to the differences. Much of the substructure in these plots is due to the distinct satellite galaxies in the simulation (see Nickerson et al. 2011) rather than the diffuse halo, which are usually excluded from observational studies (e.g., Bell et al. 2008). We therefore focus on the righthand plots, in which bound substructures have been removed, and particularly on the bottom-right panel, where the ellipsoidal shape of the halo has been taken into account.

The number of particles could impact the amount of structure, and certainly the error bars, complicating the comparison between the SPH-STARS and SPH-PAINTED halos. We therefore first compare the SPH-STARS (black) and SPH-STARS (LOWSAMP; green) halos and find that the error bars are indeed significantly larger at lower resolution, but that the results always agree to within the error bars, giving us confidence that the error bars accurately portray the uncertainty in the measurement. Although it appears that the subsampled halo is systematically less structured, this is a coincidence of the randomly sampled particles that constitute the LOWSAMP halo; with different random seeds, the LOWSAMP halo scatters both positive and negative around the full resolution halo, with a dispersion comparable to the quoted error bar. We therefore conclude that differences between halo models that are larger than the error bars are real discrepancies between the predictions.

We assess the impact of using painted DM particles instead of stars by comparing SPH-STARS (black) to SPH-PAINTED (red). SPH-PAINTED is systematically less structured at all radii when using our preferred ellipsoidal bins, typically by a factor of two. There is little difference between the dispersion measured in spherical versus ellipsoidal shells, which is consistent with the similar global shapes of these halos (Figure 5). The SPHEXACT halo (magenta) is indistinguishable from the SPHPAINTED halo, indicating that the difference in the amount of substructure is entirely due to the use of painted DM particles.

The impact of the different potential in the baryonic simulations can be seen by comparing SPH-PAINTED (red) to DMPAINTED (blue). The DM-only simulation is systematically more structured at all radii, in this case by factors of typically 3 , although as high as 7 at intermediate radii. Although the global halo shapes are different enough that they could introduce a significant dispersion in a structureless halo, the same discrepancy is seen when using spherical shells at almost all radii, allowing us to be confident that this is not an artifact of the more flattened halo in the DM-only simulation. 


\section{DISCUSSION}

We have tested the effects of two common assumptions used in stellar halo models: (1) that stellar mass can be painted onto DM particles, and (2) that the baryonic changes in the potential can be ignored. We discuss below the effects of each assumption.

\subsection{Painting}

When we compare the SPH-STARS and SPH-PAINTED halo models, we find that SPH-PAINTED is less concentrated and less structured than SPH-STARS. Both models put, on average, the same stellar mass into the same progenitor objects and evolve them in the same potential, so it is surprising that there is such a large difference. This difference is entirely due to (1) assuming that the scatter in the stellar-DM mass relation does not correlate with the fate of the accreted halo, and (2) assuming that DM particles deep in the potential well of a subhalo (note that we only paint the most bound $1 \%$ of the DM particles) evolve similarly to the star particles, which are also found deep in the potential well. The SPH-EXACT halo, where the scatter is not an issue, is slightly more concentrated than SPH-PAINTED, indicating there is a slight tendency for halos with high stellar masses to wind up in the inner parts of the halo, but that this effect does not dominate the overall radial profile. Moreover, SPH-EXACT and SPH-PAINTED have identical substructure, indicating that the main reason that the SPH-STARS and SPHPAINTED models differ is because DM particles deep in the potential well are different from stars deep in the potential well. Although these particles are co-located, the kinematics of the populations are not identical: the DM particles are dynamically hotter, having orbits that take them further from the center of the subhalo (a consequence of the more extended nature of the DM component). We postulate that this difference causes the DM particles to be stripped earlier than stars during the subhalo's accretion and orbit around the parent galaxy, and results in a less concentrated halo. The higher velocity dispersion of the DM also means the stripped debris is less coherent, resulting in less substructure. In both cases the debris orbits in the same potential, and it is therefore not surprising that painting does not affect the shape of the predicted stellar halo.

It is important to note that we have formally only tested one particular painting scheme, and this scheme is less sophisticated than many that are used. Therefore, we must be careful about what lessons are generalizable.

First, we note that it is a generic property of galaxy formation physics that baryons are more concentrated than DM within subhalos, and as a direct consequence the DM particles at the same radii as star particles have different kinematic properties such as velocity dispersions and angular momenta. We therefore expect these results to generalize to any painting scheme that does not explicitly guarantee that the painted DM particles share not only the same spatial distribution as the stars within satellite galaxies, but also the same kinematic distribution.

Second, it appears that the systematic difference that painting induces in the halo concentration can completely explain discrepancies in the literature between the radial density profiles in the models of BJ05, which used painting, and the accreted stellar halo in the hydro simulations of L11, which did not.

L11 advocate one particular painting scheme where DM particles are chosen to lie within a given depth of the subhalo potential well, and demonstrate that the resulting painted stellar halo model has the same concentration as the accreted stars in their hydro simulation. This painting scheme requires higher resolution than present in our simulations, so we cannot directly test it, but we find the match between the concentration of the stars and painted DM particles very encouraging; testing to see if the painted DM halo has the same degree of substructure as the star particles is another critical test that we would strongly advocate. However, there is one important aspect of this scheme that may compromise its use for stellar halo models: the scheme was calibrated so that the radial distribution of the diffuse halo, i.e., the stripped satellites, matched the SPH stars ${ }^{8}$ without regard to the properties of the satellites themselves. There is therefore no guarantee that the stellar masses (and consequently metallicities; Tremonti et al. 2004) or radial profiles of the satellites are correct, and in fact, scaling arguments suggest that the stellar masses, in particular, may not scale correctly with total DM halo mass. In other words, it may be that L11 are building a realistic-looking halo out of the wrong pieces; if this is true, then properties like the metallicity structure, which is one of the key observables one would like to extract from stellar halo models (e.g., Font et al. 2006), will be incorrect. Reproducing the properties of the satellites themselves is a critical test that any painting scheme must pass, and it is not immediately obvious whether it is possible for any method of painting DM particles to satisfy all of the necessary constraints.

The most important and general point is that painting can easily introduce systematics at the factor-of-several level. We strongly urge modelers to perform tests like these to estimate the magnitude of the systematic error when introducing new painting schemes.

\subsection{Baryonic Potential}

When comparing the SPH-PAINTED and DM-PAINTED halo models, we find that DM-PAINTED is less concentrated, its shape is more prolate and less flattened, and it has more substructure. Both models use the same painting scheme on the same progenitors, but evolve them in a different gravitational potential. The critical differences between the potentials are that the SPH-PAINTED model has a parent galaxy that is more concentrated and has a disk, and also has satellites with deeper potential wells.

The global shape of the potential clearly has an impact on the global shape of the stellar halo: when the potential is more concentrated, so is the stellar halo; when the potential is more spherical, so is the stellar halo; when the potential is more prolate, so is the stellar halo. The baryons also make it more difficult to strip particles out of the satellites, so the satellites must get closer to the center to get stripped and therefore deposit their tidal debris closer to the center.

The reason for the different amounts of substructure is less clear. There are two physical mechanisms that could decrease the amount of substructure in SPH-PAINTED: differential precession of streams in the oblate potential of the disk, and changes in individual orbits due to the central concentration of the potential (below we will refer to this as "scattering" for simplicity, although for an extended central concentration, like a disk, this is primarily due to individual orbital types changing their shape rather than true scattering onto chaotic orbits that is seen for point-like central concentrations; Debattista et al. 2008; Valluri et al. 2010). We do not have the ability to independently assess

\footnotetext{
8 Note that although the scheme was calibrated to recover the correct radial distribution, the fact that it was able to do so via tuning one parameter is not a trivial result: as we have demonstrated, many other painting schemes are not able to do this for any choice of parameters.
} 
each effect with this simulation, but we note that the global potential in the DM-only simulation is more prolate-triaxial than in the SPH simulation, and it is only in the disk region that the SPH potential has a significantly smaller $c / a$ axis ratio. We may therefore expect differential precession to disrupt streams in the outer parts of the halo faster in the DM-PAINTED model but to disrupt streams in the inner parts of the halo faster in the SPH-PAINTED model. Instead, the SPH-PAINTED model has less structure at all radii, but the effect is indeed strongest within $40 \mathrm{kpc}$. This suggests that both differential precession and orbital scattering are playing a role.

The overproduction of substructure in pure DM models must be taken into account when comparing these models to observations. For example, Helmi et al. (2011) determine that the Milky Way halo contains less structure than predicted by the C10 pure DM models, and conclude that the Milky Way halo must also contain a smooth in situ component that reduces the total substructure. However, the factor by which Helmi et al. (2011) find that the model overpredicts the structure is 2-3, which is of the same magnitude as the level of systematic overprediction of substructure we find for pure DM models. We therefore urge caution against overinterpreting differences between these models and observations that are smaller than the scale of the systematics that we find.

\subsection{Simulation Caveats}

Because the galaxy in the SPH simulation is not a perfect representation of a real galaxy, it is worth discussing how those differences might affect our conclusions.

The primary differences between the simulated galaxy and a typical disk galaxy of the same mass are that (1) its potential is too concentrated and (2) its disk fraction is too small. We therefore might expect that effects we see that are due to the concentration of the potential might be overestimated, while those due to the diskiness of the potential might be underestimated.

We argue that the systematic error due to painting primarily arises because the kinematics of DM particles deep in the potential of a satellite are different from those of stars deep in the potential. In a more realistic less concentrated potential, the kinematic differences between different particle types might be expected to be less, suggesting an overestimate of the level of this systematic effect. On the other hand, if the stellar distribution were diskier, the kinematics of the stars in that rotating disk would differ even more from those of the nondisky DM particles, suggesting we are underestimating the level of this systematic. We do not know a priori which of these effects would dominate, so the quantitative degree of the effect is uncertain, but the overall sign and approximate magnitude of the systematic are likely to be faithfully indicated by this work.

The SPH-PAINTED stellar halo is more concentrated than the DM-PAINTED stellar halo due to the concentration of the baryonic potential. Because the baryonic potential is too concentrated in the SPH simulation, the degree to which the baryons concentrate the stellar halo could also be overestimated.

The amount of substructure in a more realistic potential would be expected both to increase because of the less concentrated potential and decrease because of the diskier potential-we believe both phenomena are important in determining the amount of substructure, as discussed in Section 5.2. Again, this may affect the quantitative measurement but is unlikely to affect the sign or overall magnitude of the systematic effect, which are our main conclusions.
One further caveat is that the dynamical mass of star particles in the SPH-STARS model is significantly smaller than the mass of the DM particles, so the central region of the SPH simulation, where the stars dominate, is effectively simulated at higher resolution than in the DM-only simulation. One could therefore imagine that some of the differences we see are related to resolution rather than to baryonic effects. However, it is unlikely that this would drive our results. Increasing the mass resolution of a DM-only simulation, as we would need to do to match it to the mass resolution of the SPH stars, does not result in different structure at the scales of interest: higher-resolution DM halos are comparably triaxial, have comparable radial profiles, and have comparable numbers of higher-mass (i.e., resolved in the lower-resolution simulation) subhalos (e.g., Stadel et al. 2009; Gao et al. 2011). A related issue, however, is that hydrodynamic simulations are much more sensitive to resolution, and so the detailed structure of our SPH galaxy and therefore of the stellar halo models built from it could be different than that of a galaxy simulated at higher resolution. However, the main reasons that we see a difference are because the baryons form a concentrated distribution, and because that distribution is disk-shaped; both of these facts will be true of any hydrodynamic simulation that forms a disk galaxy. Therefore, while the detailed structure of the halo model may differ at different resolutions, the comparison between the halo models should still yield a good estimate for the magnitude of the systematic effects that are introduced by the modeling assumptions.

Overall, we therefore caution that the differences between the simulated galaxy and real galaxies may have a quantitative effect on our results. However, the sign and approximate magnitude of the systematic differences reported here are expected to be robust, with the possible exception of the large differences in concentrations between the SPH halo and the DM-only case, which is expected to persist qualitatively but quantitatively may be substantially overestimated in a simulation such as ours with an overly concentrated baryonic component.

\section{CONCLUSIONS}

We have examined two critical assumptions that are part of most models of the structure of stellar halos: using painted DM particles to represent stars, and the omission of baryonic contributions to the gravitational potential. We have used a controlled set of models where we can independently test their effects. In one test, we compare stars formed in a cosmological hydro simulation to painted DM particles in the same simulation to test the effect of painting, while in the other test we compare DM particles painted in a hydro simulation to DM particles painted in the identical way in a pure $N$-body realization of the same initial conditions to test the effect of the different potentials.

We find that both sets of assumptions cause significant differences in the properties of the predicted stellar halos. Painting results in a less concentrated halo, with a half-mass radius $\sim 6$ times larger, and a less structured halo, by a factor of $\sim 2$. Some of the concentration difference is due to a systematic tendency for progenitors with high stellar mass to wind up at small radius, but most of the concentration difference and all of the structural difference is due to the different kinematics of DM particles and stars at the same location within a satellite. The omission of the baryonic contribution to the potential results in a halo that is less concentrated, by a factor of 1.7 in half-mass radius, more structured, by a factor of 2-7, and more prolate, with $b / a \sim c / a \sim 0.6$. The mechanisms that drive 
these changes are likely a combination of orbit scattering from the central density enhancement, differential precession when orbits are near the disk, and the overall prolateness of the DM halo.

This is the first attempt we are aware of to estimate the magnitude of the systematic effects present in stellar halo models based on DM simulations, and the results are somewhat discouraging. The factor-of-a-few level of systematic uncertainty is similar to the factor by which some of the models are discrepant from observations of the Milky Way halo, meaning we cannot presently conclude anything about the origin of the halo from that discrepancy.

The hydrodynamic simulations differ from observed galaxies in some important ways that could affect our results; most importantly, the baryons in the simulation are more concentrated, and the baryonic distribution is less disky. These could affect the quantitative measurements that we make, but because these two differences act in opposite directions, the overall sign and approximate magnitude of the systematic error we measure is unlikely to be affected (with the possible exception of the halo concentration, which could potentially be substantially overestimated). We also caution that parts of the simulations we are comparing operate at different resolution due to the presence of low-mass star particles; however, we have argued that this is unlikely to undermine our conclusions.

Are there methods that can potentially be used to create high resolution stellar halo models that circumvent these difficulties? One possible way forward, which we are pursuing, is to couple a very sophisticated painting technique to an evolving halo potential that both self-consistently solves the DM dynamics and includes an analytic disk (which should itself be consistent with the properties of a galaxy forming within that DM halo, for example via a semi-analytic model). We stress that it is important for any painting technique to be tested, using methods such as the one we have used in this paper, to ensure that it reproduces the spatial distribution and substructure expected from stars that form with the same efficiency, and that it reproduces the properties (such as stellar mass fraction) of the satellite galaxies. Such a hybrid approach is likely to provide the best hope for modeling stellar halos in the near term. Looking forward, the highest resolution hydro simulations today are beginning to be able to produce interesting predictions, and future hydro simulations with hundreds of millions of particles within the virial radius are likely to provide the best models once they are feasible.

We thank the referee, Joop Schaye, for a helpful report. This work has made use of the Shared Hierarchical Academic Research Computing Network (SHARCNET) Dedicated Resource Project: "MUGS: The McMaster Unbiased Galaxy Simulations Project" (DR316, DR401 and DR437). This work was supported in part by National Science Foundation grant AST 1008324. M.V. was supported by National Science Foundation grant AST0908346. V.P.D. is supported by STFC Consolidated grant No. $\mathrm{ST} / \mathrm{J} 001341 / 1$. G.S. received funding from the European Research Council under the European Union's Seventh Framework Programme (FP 7) ERC Grant Agreement No. [321035]. H.M.P.C. and J.W. acknowledge financial support from the National Science and Engineering Research Council.

\section{REFERENCES}

Bailin, J., Bell, E. F., Chappell, S. N., Radburn-Smith, D. J., \& de Jong, R. S. 2011, ApJ, 736, 24

Bailin, J., Kawata, D., Gibson, B. K., et al. 2005, ApJL, 627, L17

Bell, E. F., Xue, X. X., Rix, H., Ruhland, C., \& Hogg, D. W. 2010, AJ, 140,1850

Bell, E. F., Zucker, D. B., Belokurov, V., et al. 2008, ApJ, 680, 295

Bullock, J. S., \& Johnston, K. V. 2005, ApJ, 635, 931 (BJ05)

Cooper, A. P., Cole, S., Frenk, C. S., et al. 2010, MNRAS, 406, 744 (C10)

Debattista, V. P., Moore, B., Quinn, T., et al. 2008, ApJ, 681, 1076

Font, A. S., Johnston, K. V., Bullock, J. S., \& Robertson, B. E. 2006, ApJ, 638,585

Font, A. S., McCarthy, I. G., Crain, R. A., et al. 2011, MNRAS, 416, 2802

Gao, L., Frenk, C. S., Boylan-Kolchin, M., et al. 2011, MNRAS, 410, 2309

Helmi, A., Cooper, A. P., White, S. D. M., et al. 2011, ApJL, 733, L7

Ibata, R. A., Lewis, G. F., McConnachie, A. W., et al. 2014, ApJ, 780, 128

Kazantzidis, S., Bullock, J. S., Zentner, A. R., Kravtsov, A. V., \& Moustakas, L. A. 2008, ApJ, 688, 254

Kazantzidis, S., Kravtsov, A. V., Zentner, A. R., et al. 2004, ApJL, 611, L73

Knebe, A., \& Wießner, V. 2006, PASA, 23, 125

Knollmann, S. R., \& Knebe, A. 2009, ApJS, 182, 608

Koposov, S. E., Yoo, J., Rix, H.-W., et al. 2009, ApJ, 696, 2179

Kravtsov, A. 2010, AdAst, 2010, 281913

Libeskind, N. I., Knebe, A., Hoffman, Y., Gottlöber, S., \& Yepes, G. 2011, MNRAS, 418, 336 (L11)

Majewski, S. R., Skrutskie, M. F., Weinberg, M. D., \& Ostheimer, J. C. 2003, ApJ, 599, 1082

McConnachie, A. W., Irwin, M. J., Ibata, R. A., et al. 2009, Natur, 461, 66

Monachesi, A., Bell, E. F., Radburn-Smith, D. J., et al. 2013, ApJ, 766, 106

Nickerson, S., Stinson, G., Couchman, H. M. P., Bailin, J., \& Wadsley, J. 2011, MNRAS, 415, 257

Peñarrubia, J., Benson, A. J., Walker, M. G., et al. 2010, MNRAS, 406, 1290

Purcell, C. W., Bullock, J. S., \& Zentner, A. R. 2007, ApJ, 666, 20

Rashkov, V., Madau, P., Kuhlen, M., \& Diemand, J. 2012, ApJ, 745, 142

Schlaufman, K. C., Rockosi, C. M., Lee, Y. S., et al. 2012, ApJ, 749, 77

Spergel, D. N., Bean, R., Doré, O., et al. 2007, ApJS, 170, 377

Stadel, J., Potter, D., Moore, B., et al. 2009, MNRAS, 398, L21

Stinson, G. S., Bailin, J., Couchman, H., et al. 2010, MNRAS, 408, 812

Tasca, L. A. M., \& White, S. D. M. 2011, A\&A, 530, A106

Tissera, P. B., Scannapieco, C., Beers, T. C., \& Carollo, D. 2013, MNRAS, 432, 3391

Tissera, P. B., White, S. D. M., Pedrosa, S., \& Scannapieco, C. 2010, MNRAS, 406, 922

Tremonti, C. A., Heckman, T. M., Kauffmann, G., et al. 2004, ApJ, 613, 898

Valluri, M., Debattista, V. P., Quinn, T., \& Moore, B. 2010, MNRAS, 403,525

Xue, X.-X., Rix, H.-W., Yanny, B., et al. 2011, ApJ, 738, 79

Zemp, M., Gnedin, O. Y., Gnedin, N. Y., \& Kravtsov, A. V. 2011, ApJS, 197,30

Zolotov, A., Willman, B., Brooks, A. M., et al. 2009, ApJ, 702, 1058 